\title{
Who's afraid of the big bad wolf?
}

\author{
From the ${ }^{\mathrm{a} D e p a r t m e n t}$ of Cardiothoracic Surgery, Pinnacle Health, Harrisburg, Pa; ${ }^{\mathrm{b}}$ Department of Cardiac Sur- \\ gery, Heart Hospital Baylor Plano, Baylor Scott \& White Health, Dallas; and ${ }^{\mathrm{c} D e p a r t m e n t ~ o f ~ C a r d i o v a s c u l a r ~}$ \\ Surgery, Houston Methodist DeBakey Heart \& Vascular Center, Houston, Tex. \\ Received for publication Jan 18, 2017; accepted for publication Feb 13, 2017; available ahead of print March 23, \\ 2017. \\ Address for reprints: Michael J. Reardon, MD, 6550 Fannin, Suite 1401, Houston, TX 77030 (E-mail: mreardon@ \\ houstonmethodist.org). \\ J Thorac Cardiovasc Surg 2017;153:1287-9 \\ $0022-5223 / \$ 36.00$ \\ Copyright (C) 2017 by The American Association for Thoracic Surgery \\ http://dx.doi.org/10.1016/j.jtcvs.2017.02.035
}

Mubashir Mumtaz, MD, ${ }^{a}$ Hemal Gada, MD, ${ }^{a}$ Michael J. Mack, MD, ${ }^{b}$ and Michael J. Reardon, MD

Cardiac surgeons traditionally have been leaders in the development of innovative therapy for the treatment of cardiovascular diseases. We hold deep convictions and strong opinions on discovering and delivering the best treatment for our patients. We have led the way in all of medicine in data collection and rigorous outcomes analysis to improve the care of our patients. Our passion for rigorous self-examination is unparalleled. Simply put, we are staunch patient advocates who do not run from the challenges of finding and delivering the best care to our patients.

Surgical aortic valve replacement (SAVR) for the treatment of symptomatic severe aortic stenosis is arguably one of the most successful cardiac procedures ever developed. Since the introduction of SAVR in 1960, millions of lives have been saved and improved. Although never tested against the previous standard of care, medical therapy, we as surgeons knew it was the right choice because without SAVR these patients were resigned to heart failure and death. We did track our outcomes both individually and nationally by the creation of the Society of Thoracic Surgery Adult Cardiac Surgery database, which now encompasses more than 6.1 million patients contributed by more than $95 \%$ of cardiac surgeons in the United States.

Cardiac surgeons have faced previous challenges to our role in treating cardiovascular diseases. When catheterbased coronary interventions such as percutaneous coronary intervention began in 1977, many cardiac surgeons worried about the rapid adoption of this competitive revascularization procedure without a robust evidence base for comparative effectiveness to coronary artery bypass grafting. We, in an equal leadership role with our cardiology colleagues, eventually ran a series of randomized control trials (RCTs), including the Coronary Artery Surgery Study, the Arterial Revascularization Therapies Study, the Synergy Between PCI With TAXUS

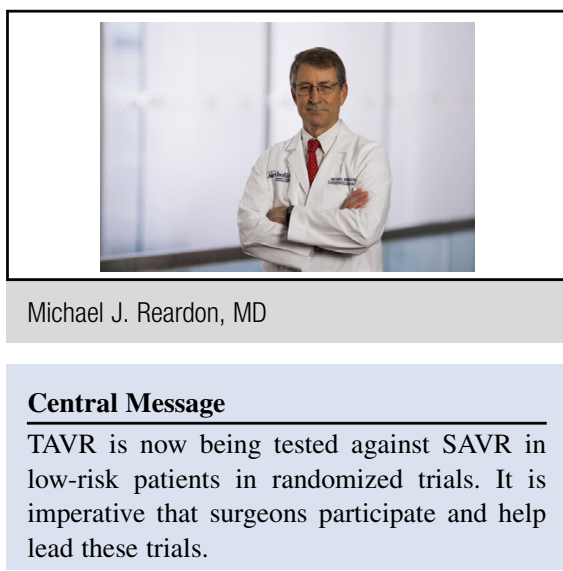

See Editorial Commentary page 1290.

Drug-Eluting Stent and Cardiac Surgery (SYNTAX), and most recently the Evaluation of XIENCE PRIME Everolimus Eluting Stent System (EECSS) or XIENCE V EECSS Versus Coronary Artery Bypass Surgery for Effectiveness of Left Main Revascularization (EXCEL), between coronary artery bypass grafting and percutaneous coronary intervention. These RCTs provided the highest level of evidence that is reflected in our guidelines and helped both cardiac surgeons and cardiologists define the best and most appropriate treatment for our patients. Despite coronary artery bypass being the most common cardiac surgical procedure, $69 \%$ of all cardiac operations in 2016, we did not fear or run from participating in these randomized trials or shrink from our responsibility to create a data-driven approach to clinical decisionmaking. In short, we were willing to let the data speak for itself. The National Heart Lung and Blood Institutesponsored Cardiothoracic Surgery Network randomized trial of mitral valve repair versus replacement in secondary mitral regurgitation recently challenged a long-held belief that repair is always better than replacement. Once again, cardiac surgeons welcomed the challenge of this randomized trial in an effort to obtain the best information possible to guide us in the care of our patients. Now, once again, cardiac surgeons are leading the way in the randomized transcatheter aortic valve replacement (TAVR) versus SAVR trials in the United States.

Unlike catheter-based coronary interventions that were initially introduced clinically into the lowest-risk patients and gradually moved to more complex and higher-risk patients, TAVR was introduced into patients who are 
often not candidates for SAVR because they were considered too high of a surgical risk by experienced surgeons on the basis of their Society of Thoracic Surgery predicted risk of mortality and clinical judgment. Subsequently, 2 RCTs of TAVR versus SAVR in highrisk patients with symptomatic severe aortic stenosis were performed in the United States. The Placement of Aortic Transcatheter Valves (PARTNER) IA and the CoreValve high-risk randomized trials both showed results considered equivalent (noninferior) or better (superiority) than SAVR that led to Food and Drug Administration (FDA) approval for the balloonexpandable and self-expanding valves in this patient population and led to an updating of our guidelines. The randomized trial for the balloon-expandable TAVR valve in intermediate-risk patients, PARTNER IIA, yielded similar results to SAVR and led to FDA approval of this valve for treatment of the intermediate-risk population. The Surgical Replacement and Transcatheter Aortic Valve Implantation was presented at the American College of cardiology 2017 and showed equivalent results to surgery. In short, more than 3500 patients have been enrolled in randomized trials comparing the 2 procedures in the United States, leading to a robust evidence base.

Cardiac surgeons were on the front lines of all of these trials with equal participation in trial leadership and largely drove enrollment as arbiters of risk. They not only actively participated in the design, enrollment, analysis, and implementation of these trials with a free and unimpeded voice, but also played a key role in presenting these results in late-breaking clinical trials sessions at society meetings and in front of the FDA to obtain the eventual approval of these devices.

Because high-risk patients constitute approximately $8 \%$ of the population currently undergoing SAVR and the intermediate risk is approximately $12 \%$, cardiac surgeons did not feel threatened to enroll high-risk patients and had a reasonably low level of anxiety about intermediate-risk patients because they thought that creating the strong evidence was crucial and that it had a minimal impact on affecting their clinical practices. These trials have yielded some of the most robust and valuable data available in the world of cardiac valve disease treatment. After these landmark trials, we now have 2 low-risk randomized trials actively enrolling approximately 2400 patients in the United States: the PARTNER 3 and the Evolut LowRisk trial. Surgeons are now being asked to support randomizing the bulk of their patients currently undergoing a SAVR.

For many of us, it is clear that TAVR is here to stay and likely to continue to expand its indications. Whenever the outcomes of 2 therapies are equivalent, patients and their referring physicians usually prefer the lessinvasive therapy. It is not likely that we will be able to "protect our turf" short of robust randomized data that show SAVR superior to TAVR or long-term durability data on TAVR that are worse than on SAVR. Therefore, the big question is, are we as surgeons going to embrace this technology and actively participate in and guide this process or leave this to others? Unfortunately, the indications are otherwise, because the enrollment cadence in the low-risk trials has been slower than expected from previous trials of TAVR versus SAVR. Make no mistake, the low-risk trial is a surgeon-driven trial because the majority of these patients are seen in our offices on a daily basis. As surgeons, we believe it is our obligation to inform and educate these patients. We need to explain all available options, including enrollment in these trials, to the patients and their families. Although we currently do not know the outcomes in low-risk patients, all previous highly reliable and well-conducted randomized trials have shown equivalence in some or superiority in others for TAVR in the higher-risk group of patients. Even the skeptics among us would have to admit that there is a chance that this therapy may be better than the current gold standard and that it is our responsibility to incorporate this in our discussions and seek an answer.

We as surgeons rightly often bring durability of TAVR into question because long-term data are not available. Five-year follow-up of TAVR has actually shown excellent freedom from structural valve degeneration with no signal of premature structural valve degeneration, and as we follow these patients further we will know more. The surgical valve durability has not been proven in a prospective well-conducted study using echocardiogram and computed tomography to assess degeneration. In fact, even the best available data for surgical valve durability has been based on retrospective registries, only available for some valves, with less than ideal follow-up, limited echocardiography analysis, and no computed tomography analysis. These studies generally define structural valve degeneration on the basis of the need for reoperation, which is a crude way of looking at it. Both low-risk trials have committed to following all treated patients, both SAVR and TAVR, for 10 years. Let's not lose this opportunity to learn about the durability of both TAVR and SAVR valves in well-controlled studies with complete long-term follow up. Now is not the time to lose our academic courage and run from participating in these trials.

If we don't embrace this technology now and it turns out to be better, then we run the risk of being viewed as obstructionists and choking off the prosperous future for our specialty. One only need to look as far as vascular 
surgery to see the results of embracing disruptive technology. Endovascular procedures and thus vascular cases were slowly being taken from vascular surgery as a field. When endovascular aneurysm repair became available, forward-thinking vascular surgeons knew the right thing was to adopt and lead this new area. Consequently, our vascular colleagues are now busier than they have ever been. TAVR is our endovascular aneurysm repair moment. Cardiac surgeons need to embrace, lead, and define the field of transcatheter cardiac valve therapy. To do this, we must lead in the TAVR trials, and in the current setting that means actively participating in the low-risk trial.

If we build our home with sturdy bricks of solid data, we will be safe. No amount of huffing and puffing should dissuade us. When asked, "Who's afraid of the big bad wolf?" our honest answer will be "Not us!"

\section{Conflict of Interest Statement}

M.M. reports consultant, faculty, honoraria, and research from Abbott; consultant, faculty, honoraria, and research from Atricure; member cardiac events committee USPella trial for Abiomed; consultant, faculty, honoraria, and research from Edwards; consultant, faculty, honoraria, and research from Medtronic; cardiac events committee for Millipede; instructor, honoraria from JOMDD; and research for Keystone heart. H.G. reports consultant, faculty, honoraria, and research from Abbott; consultant, faculty, honoraria, and research from Edwards; consultant, faculty, honoraria, and research from Medtronic; and research from Keystone Heart. M.J.M. is the national surgical Principal Investigator for the Edwards PARTNER 3 trial. M.J.R. is the national surgical Principal Investigator for the Medtronic low-risk randomized trial. 